\title{
Belphégor
}

\section{Zombie Theory, a Reader, sous la direction de Sarah Juliet Lauro}

\section{Julien Martins}

\section{(2) OpenEdition \\ 1 Journals}

\section{Electronic version}

URL: https://journals.openedition.org/belphegor/1162

DOI: 10.4000/belphegor.1162

ISSN: 1499-7185

Publisher

LPCM

\section{Electronic reference}

Julien Martins, "Zombie Theory, a Reader, sous la direction de Sarah Juliet Lauro", Belphégor [Online],

16-1 | 2018, Online since 04 July 2018, connection on 19 October 2021. URL: http://

journals.openedition.org/belphegor/1162 ; DOI: https://doi.org/10.4000/belphegor.1162

This text was automatically generated on 19 October 2021

\section{(c) (i) (9)}

Belphégor est mis à disposition selon les termes de la Licence Creative Commons Attribution - Pas d'Utilisation Commerciale - Pas de Modification 4.0 International. 


\section{Zombie Theory, a Reader, sous la direction de Sarah Juliet Lauro}

Julien Martins

\section{REFERENCES}

Zombie Theory, a Reader, sous la direction de Sarah Juliet Lauro, University of Minnesota Press

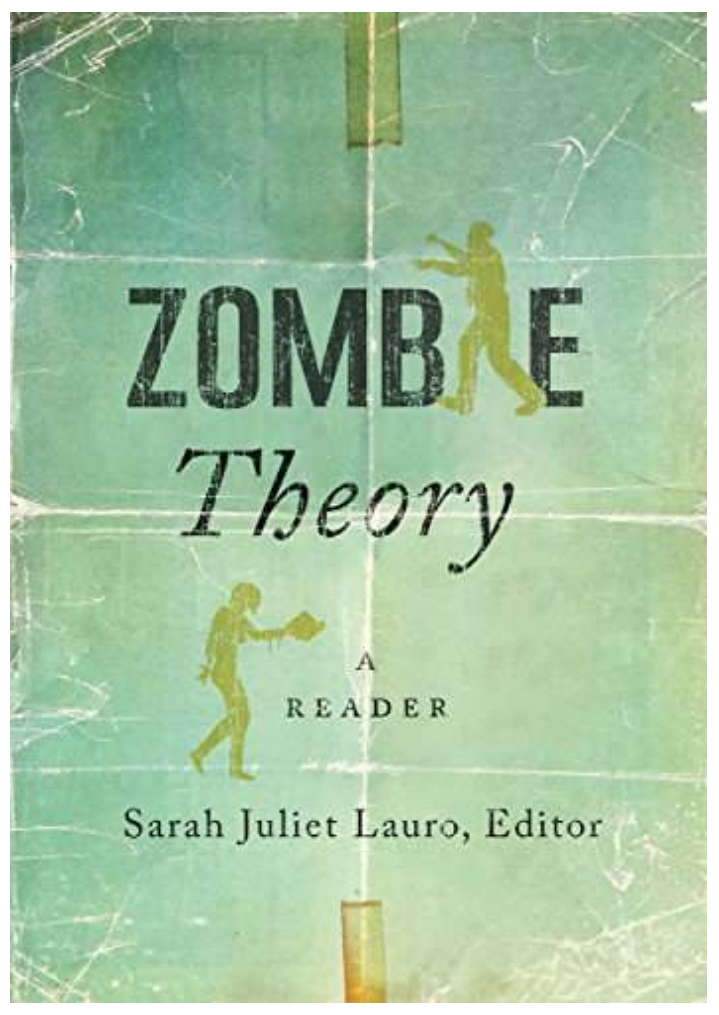


1 Depuis la seconde moitié du vingtième siècle, la figure du zombie a envahi la culture populaire. Entre Night of the Living Dead de Romero (1968) et la très récente série The Walking Dead, le zombie s'est propagé et a connu de nombreuses transformations. S'il est presque toujours cette créature anthropophage, dénuée de conscience et animée par un insatiable appétit, les questions que soulèvent son apparition varient. La façon dont il brouille les limites physiques et conceptuelles entre la vie et la mort intrigue autant que son impact sur une société qu'il bouscule et force à se reconstruire. Étudier la figure du zombie dans la culture populaire suppose ainsi de parcourir un vaste ensemble de productions culturelles et nécessite la prise en compte d'interrogations ontologiques, géographiques et sociales. C'est donc la diversité de ces aspects que se propose d'explorer Zombie Theory: a Reader, une anthologie d'articles consacrés au sujet, publiée en 2017 chez University of Minnesota Press et dirigée par Sarah Juliet Lauro.

2 L'ouvrage est divisé en cinq parties: «Old School, Classic Zombie»; «Capitalist Monsters »; «Zombies and other(ed) People »; «Zombies in the Street » et « New Life for the Undead ${ }^{1}$ ». Zombie theory fait alors découvrir à son lecteur l'évolution de la figure du zombie depuis les origines haïtiennes du mythe (et des problématiques raciales liées à celles-ci) jusqu'à ses manifestations les plus contemporaines (comme la bande-dessinée The Walking Dead ou encore l'utilisation du zombie dans le cadre d'une théorie post-humaniste). Le mythe du zombie est en outre aussi bien abordé en tant que critique du capitalisme qu'en tant qu'élément déclencheur d'une restructuration de l'espace géographique, politique et social.

Le zombie est une figure protéiforme et chacun des articles de Zombie Theory s'évertue à le démontrer avec un certain succès. On y notera la présence de fragments de Zombieindex ${ }^{2}$, œuvre numérique et composite de l'artiste californien George Pfau. Ces fragments parcourent l'ensemble du livre et se retrouvent en tête de chaque partie, laissant au lecteur le loisir d'explorer la diversité d'une horde de morts-vivants en apparence uniforme. Le choix d'illustrer ses différentes parties par un extrait de Zombieindex témoigne des ambitions de cette collection d'articles: offrir un vaste panorama de l'une des figures mythiques les plus représentées dans la culture populaire contemporaine et servir de point d'entrée dans son étude. Si la totalité des thématiques abordées se retrouve relativement bien organisée au sein des cinq parties de l'ouvrage et si cette organisation chronologique s'avère efficace, elle n'empêche pas le lecteur de se perdre un peu face à la multiplicité des champs évoqués.

De ces articles ressortent cependant certaines questions majeures: le zombie, par ses origines, s'inscrit d'abord dans une dialectique du maître et de l'esclave. Il est au départ un corps sans esprit, un cadavre reconverti en travailleur agricole pour le compte d'un sorcier. Reproduisant le statut des esclaves dans les colonies, il est aussi, lorsqu'il se retourne contre ses maîtres, le symbole d'une révolte, d'un bouleversement des hiérarchies sociales en vigueur, comme l'explique Elizabeth McAlister dans son article "Slaves, Cannibals, and Infected Hyper-Whites ${ }^{3}$ ». Dès lors, le zombie porte en lui des enjeux raciaux essentiels. Il incarne l'esclave noir dépossédé de son identité par le colon blanc, menaçant en retour ce dernier. Le but de l'article d'Elizabeth McAlister est en somme de montrer que les récits autour du zombie ont pour objectif $d^{\prime}$ ' interroger les limites entre la vie et la mort, d'élucider la relation complexe entre la liberté et l'esclavage et de mettre en lumière le chevauchement entre capitalisme et cannibalisme $e^{4} »$ - ces éléments ayant tous pour « pivot » la notion de race. 
Il semble ainsi naturel que, quelques décennies plus tard, ce même zombie parvienne à traduire les craintes suscitées après la Seconde Guerre mondiale par le développement d'une économie globalisée. Le zombie se voit notamment reformulé en consommateur avide, errant, en souvenir de sa vie précédente, dans les rayons d'un supermarché (dans Dawn of the living dead de Romero). Ici, explique Steven Shaviro dans son article "Contagious Allegories ${ }^{5}$ ", les zombies sont majoritairement présentés comme des «doubles ayant fonction de simulacres ». Pour résumer, «ils sont des équivalents humains plutôt que des figures opposées ${ }^{6}$ » et retranscrivent dans leur comportement frénétique et leurs déambulations la «consommation, par la classe moyenne américaines, de biens inutiles ${ }^{7}$ ». De cette manière inscrits dans un système porté d'une part par la consommation excessive et de l'autre par l'optimisation permanente de la production servant à la soutenir, les zombies cinématographiques, cadavres ambulants privés de libre arbitre, renvoient au spectateur l'image familière du travailleur des classes moyennes.

6 Le zombie, enfin, dans son état de "vie nue ${ }^{8}$ ", libéré des entraves de la subjectivité, réduit à sa fonction première d'organisme plus ou moins vivant, offre un modèle complexe aux théories post-humanistes. Sarah Juliet Lauro et Karen Embry, dans leur article «A Zombie Manifesto, The Nonhuman Condition in the Era of Advanced Capitalism ${ }^{9}$ », nourri du «Cyborg Manifesto ${ }^{10}$ » de Donna Haraway, utilisent le zombie comme un objet théorique donnant la possibilité de penser l'être « post-humain ». Les deux auteurs reproduisent au sein de leur article le mouvement général de l'ouvrage et englobent non seulement le zombi haïtien mais aussi le zombie cinématographique contemporain ainsi qu'un dernier zombie qui, selon elles, "n'existe[rait] pas encore " ou du moins uniquement comme « une expérience de pensée exposant les limites d'une théorie posthumaine et montrant que l'on ne peut devenir posthumain qu'au [moment de la mort du sujet ${ }^{11}$ ». Le zombie illustre en effet la peur d'un corps corruptible et pesant duquel, pourtant, il est impossible de se défaire. Il réactualise alors le paradigme chair / esprit en interrogeant aussi bien le corps terrifiant que la place de la subjectivité face à l'impératif organique. Le zombie est un posthumain en ce qu'il invite justement à dépasser la division traditionnelle entre l'objet d'une part et le sujet de l'autre. Il signe en cela la mort du capitalisme puisqu'il s'attaque au principe d'identité individuelle et le met à mal au profit d'une identité collective où la séparation objet/ sujet est abolie.

7 L'article "Nekros; or, The Poetics of Biopolitics ${ }^{12}$ », d'Eugene Thacker prolonge cette réflexion sur la dimension collective du zombie en reprenant la notion de «biopolitique " telle que l'a définie Michel Foucault ${ }^{13}$, $c^{\prime}$ est à dire le point à partir duquel l'exercice du pouvoir empiète sur la vie organique. La «biopolitique » est envisagée comme la recherche d'un mode de gouvernance capable de prendre en compte ce qu'il y a de "non-humain » dans la vie, les flux qui la parcourent et qui dépassent l'individu pour s'établir dans la multitude. Il s'agit pour l'auteur de démontrer que les figures du démon puis du zombie expriment les limites a priori insurmontables d'un "bio-pouvoir». Ces deux figures concentrent selon Eugene Thacker les ponts qui s'établissent inévitablement entre médecine et théologie, science et surnaturel. La décomposition irréversible et permanente du zombie et le chaos qu'engendre l'intrusion dans l'organisme social du démon viennent, semblables à une pathologie, perturber le mouvement normal de la vie (augurant en cela des dangers guettant une société biopolitique). Zombies et démons sont en effet les hérauts d'un 
déclin du pouvoir devant l'échec d'une entreprise de régulation totale des corps et de leur circulation. Ils donnent un aperçu de la révolte potentielle de ces corps que l'on souhaite dompter et surveiller. La densité du sujet ainsi que l'importante variété des notions convoquées peuvent néanmoins rendre le propos hermétique au lecteur peu familier avec ces concepts. Le principal atout de Zombie Theory, sa pluralité et son exhaustivité, est donc en même temps son plus grand défaut.

Il est facile de se sentir désarmé face à un tel corpus d'œuvres et de s'égarer parmi toutes les pistes de lecture proposées. Les indications bibliographiques fournies par l'éditrice au terme de chacune de ses introductions compensent en partie ce défaut et permettent de guider le lecteur novice qui souhaiterait approfondir un thème spécifique. On remarque immédiatement un désir de simplifier la circulation entre les articles qui, s'ils touchent à des questions parfois fort éloignées, n'en sont pas moins explicitement connectés entre eux. Comprendre par exemple ce qu'implique le zombie dans le cadre d'un «bio-pouvoir » revient aussi à saisir les raisons pour lesquelles il faut circonscrire son invasion. Son altérité et sa multiplicité apportent avec elles une pression constante sur les frontières spatiales et politiques d'une société ne subsistant qu'à travers le contrôle strict des masses. Les articles sont par conséquent parfaitement complémentaires et parviennent à dresser une vue d'ensemble cohérente de leur objet d'étude commun.

Zombie Theory réussit à éclairer son lecteur sur les possibilités interprétatives contenues dans une figure dont l'avenir est encore plein de promesses. C'est avec succès que l'ouvrage répond à son ambition initiale. Il est une porte d'entrée idéale pour l'étude du mythe : soulignant toutes ses ramifications, il se retrouve en même temps pris dans le vertige qu'elles comportent. Ce vertige, pourtant, loin de provoquer la confusion chez le lecteur, lui est brillamment communiqué. Laissant présager des futures évolutions du mythe, Zombie Theory s'attache à produire des grilles d'analyse efficaces en retraçant son histoire et sa diffusion dans la culture populaire contemporaine. C'est un zombie aux mille visages qui y est présenté : tantôt esclave d'un patron-sorcier ou d'une société affamée, tantôt essaim, collectivité organique où la subjectivité n'a plus siège. Nul doute que d'autres visages viendront encore se greffer sur la figure du zombie et que la lecture de Zombie Theory permettra de mieux les reconnaître.

\section{NOTES}

1. Il serait possible de traduire les titres de cette manière : I- Vieille école, le zombie classique ; IIMonstres capitalistes; III- Zombies et populations ostracisées (le jeu de mot nous semblant difficilement transposable en français); IV- Zombies dans la rue ; V- Nouvelle vie pour le nonmort.

2. Consultable à cette adresse : http://zombieindex.net/

3. Partie I, chapitre 4, p.63-84.

4. " In both contexts, zombie narratives and rituals interrogate the boundary between life and death, elucidate the complex relations between freedom and slavery, and highlight the overlap between capitalism and cannibalism. » 
5. Partie I, chapitre 1, p.7-19.

6. "The zombies are overtly presented as simulacral doubles (equivalents rather than opposites) » p.12.

7. «[...] their destructive consumption of flesh [...] immediately parrallels the consumption of useless commodities by the American middle class », Sic.

8. "Bare life ", une notion théorisée par le philosophe italien Giorgio Agamben et qui renvoie à une conception de la vie où le principe purement biologique de l'existence surpasse l'expérience de celle-ci, les conditions selon lesquelles elle est vécue.

9. Partie V, chapitre 22, p.395-412.

10. Donna Haraway, «A Cyborg Manifesto: Science, Technology, and Socialist-Feminism in the Late Twentieth Century » dans Simians, Cyborgs and Women: The Reinvention of Nature (New York; Routledge, 1991), p.149-181. Pour résumer, le cyborg y est perçu à la fois comme le produit d'une réalité sociale et comme une fiction codifiée qui permettrait de lire le nécessaire dépassement des dualités traditionnelles (homme/femme, humain/animal, organique/mécanique et sujet/ objet notamment) dans le cadre d'une théorie posthumaniste et féministe. Il interrogerait la notion de conscience et par extension d'identité.

11. «[...] and finally, we are putting forth a zombie that does not yet exist : a thought experiment that exposes the limits of posthuman theory and shows that we can get posthuman only at the death of the subject. ", p.305.

12. Partie V, chapitre 20, p.361-380.

13. Dans son cours donné au Collège de France entre janvier et avril 1979 où il entendait traiter de « la manière dont on a essayé depuis le XVIIIe siècle, de rationaliser les problèmes posés à la pratique gouvernementale par les phénomènes propre à un ensemble de vivants constitués en population: santé, hygiène, natalité, longévité, races... » (dans « Naissance de la biopolitique résumé du cours au Collège de France », Dits et écrits, Vol. III, Gallimard, 1979). 